Open Access

\title{
Microglia processes associate with diffusely injured axons following mild traumatic brain injury in the micro pig
}

Audrey D. Lafrenaye ${ }^{1 *}$, Masaki Todani ${ }^{1,2}$, Susan A. Walker ${ }^{1}$ and John T. Povlishock ${ }^{1}$

\begin{abstract}
Background: Mild traumatic brain injury (mTBI) is an all too common occurrence that exacts significant personal and societal costs. The pathophysiology of $\mathrm{mTBI}$ is complex, with reports routinely correlating diffuse axonal injury (DAl) with prolonged morbidity. Progressive chronic neuroinflammation has also recently been correlated to morbidity, however, the potential association between neuroinflammatory microglia and DAl is not well understood. The majority of studies exploring neuroinflammatory responses to TBI have focused on more chronic phases of injury involving phagocytosis associated with Wallerian change. Little, however, is known regarding the neuroinflammatory response seen acutely following diffuse $\mathrm{mTBI}$ and its potential relationship to early DAl. Additionally, while inflammation is drastically different in rodents compared to humans, pigs and humans share very similar inflammatory profiles and responses.
\end{abstract}

Methods: In the current study, we employed a modified central fluid percussion model in micro pigs. Using this model of diffuse $\mathrm{mTBI}$, paired with various immunohistological endpoints, we assessed the potential association between acute thalamic DAl and neuroinflammation $6 \mathrm{~h}$ following injury.

Results: Injured micro pigs displayed substantial axonal damage reflected in the presence of APP+ proximal axonal swellings, which were particularly prominent in the thalamus. In companion, the same thalamic sites displayed extensive neuroinflammation, which was observed using Iba-1 immunohistochemistry. The physical relationship between microglia and DAl, assessed via confocal 3D analysis, revealed a dramatic increase in the number of Iba-1+ microglial processes that contacted APP+ proximal axonal swellings compared to uninjured myelinated thalamic axons in sham animals.

Conclusions: In aggregate, these studies reveal acute microglial process convergence on proximal axonal swellings undergoing DAl, an interaction not previously recognized in the literature. These findings transform our understanding of acute neuroinflammation following $\mathrm{mTB}$ and may suggest its potential as a diagnostic and/or a therapeutic target.

Keywords: Mild traumatic brain injury, Diffuse axonal injury, Neuroinflammation, Microglia, Micro pig

\footnotetext{
* Correspondence: forrestad@vcu.edu

${ }^{1}$ Department of Anatomy and Neurobiology, Virginia Commonwealth University Medical Center, P.O. Box 980709, Richmond, VA 23298, USA Full list of author information is available at the end of the article
} 


\section{Background}

Mild traumatic brain injury (mTBI) is a common insult that exacts devastating personal and social costs [1-4]. Diffuse mTBI is typically caused by accelerationdeceleration forces, such as those encountered during a motor vehicle accident or sports-related event. The forces generated by the rapid movement of the brain within the cranial vault lead to a multitude of complex metabolic, physiologic, and pathologic responses [5-9]. One pathology that frequently follows mTBI is diffuse axonal injury (DAI), in which force-induced stress results in discrete areas of scattered axonal disruption that ultimately progress to disconnection. This results in a proximal axonal segment that remains connected to the neuronal soma and a distal segment that progresses to Wallerian degeneration [10-13]. Advanced neuroimaging and histological studies have firmly established a positive correlation between DAI and TBI-induced morbidity both clinically and experimentally [10, 14-18].

In addition to DAI, progressive chronic neuroinflammation has been observed following TBI with the suggestion that this pathology is also associated to morbidity [19-24]. Neuroinflammation involves the activation of resident brain microglia and later includes systemic infiltrating macrophages in injuries that involve blood-brain barrier disruption. Upon activation/reactivation, resident microglia undergo morphological changes associated with activation, transforming from highly ramified "resting" or "surveying" phenotypes to activated microglia, with truncated processes, larger cell bodies, and less complex process networks and/or amoeboid morphologies [25-27]. Activated microglia are associated with a variety of detrimental as well as regenerative functions, including phagocytosis, cytokine secretion, and/or neurotrophin secretion [28-34]. To date, the majority of studies exploring neuroinflammatory responses to TBI, including our own, have focused on the phagocytic role of microglia in clearing Wallerian debris days following injury [29, 35-39]. Knowledge is, however, greatly lacking regarding any potential association between microglia and the proximal segment of axons undergoing DAI, independent of the degenerating distal axonal segment and/or Wallerian change.

Previously, our lab reported that while highly activated phagocytic microglia were commonly seen associated with distal degenerating axonal segments, little association was found between activated microglia and proximal axonal segments undergoing DAI [36, 39]. These studies, however, were not designed to address the aforementioned issue in that our previous studies were confined primarily to sub-acute and chronic post-injury time points. Thus, these studies provided no information regarding more acute neuroinflammatory changes or any potential relation to early DAI. Further, while our previous study utilizing transgenic mice allowed for the precise discrimination between proximal and distal axonal segments undergoing DAI, analysis was restricted to the optic nerve [36], a unique white matter region containing a homogeneous axonal population with potentially distinctive microglial responses to injury [40-42]. Lastly, as with the majority of published work in this area, our previous studies explored TBIrelated neuroinflammation in rodents, whose systemic inflammatory responses are known to differ from humans [43-46]. In consideration of the fact that pig inflammatory profiles are much more human-like $[47,48]$, we revisited the issue of microglial response to DAI acutely following injury in a micro pig model of mTBI.

Using an adapted central fluid percussion injury (cFPI) model of diffuse mTBI, we evaluated the extent of acute neuroinflammation and its relation to axons undergoing DAI in the micro pig $6 \mathrm{~h}$ following injury. Large amyloid precursor protein (APP) containing swellings, indicative of the proximal segments of axons undergoing DAI, were found diffusely scattered throughout the brain, with consistent involvement of the thalamus, an area commonly affected in human TBI [49-54]. Acute neuroinflammation accompanied these axonal changes and mapped to the same sites within the thalamus. Importantly, activated microglial processes converged on proximal axonal swellings undergoing DAI to form increased numbers of physical contacts as compared to the number of contacts made between microglia and uninjured axons in sham animals.

\section{Methods}

\section{Animals}

Experiments were conducted in accordance with the Virginia Commonwealth University institutional guidelines concerning the care and use of laboratory animals (Institutional Animal Care and Use Committee), which adhere to regulations including, but not limited to, those set forth in the "Guide for the Care and Use of Laboratory Animals: 8th Edition" (National Research Council). Twenty-one adult male Yukatan micro pigs, weighing $15-25 \mathrm{~kg}$ ( 6 months of age), were used for this study. Animals were housed in environmentally controlled pens in pairs on a 12-h light-dark cycle, with free access to food and water.

\section{Surgical preparation and injury induction}

Micro pigs were initially anesthetized with an intramuscular injection of $100 \mathrm{mg} / \mathrm{ml}$ xylazine $(2.2 \mathrm{mg} / \mathrm{kg}$; AnaSed Injection, Shenandoah, IA, USA) and $100 \mathrm{mg} /$ $\mathrm{ml}$ Telazol $(2.0 \mathrm{mg} / \mathrm{kg}$; tiletamine $\mathrm{HCL}$ and zolazepam HCL; Pfizer, New York, NY, USA) followed by intravenous administration of sodium pentobarbital $(60 \mathrm{mg} / \mathrm{kg}$; Sigma-Aldrich, St. Louis, MO, USA). Once the absence 
of a corneal reflex was verified, the micro pig was intubated and ventilated with 1-2 \% isoflurane mixed in $100 \%$ oxygen throughout the experiment. Ophthalmic lubricant (Dechra, Overland Park, KS, USA) was applied to avoid damage or drying of the eye. Body temperature was monitored with a rectal thermometer and maintained at $37{ }^{\circ} \mathrm{C}$ with a heating pad. Catheters were placed in the right femoral artery and vein for continuous monitoring of mean arterial blood pressure (MABP), assessment of blood gases, and infusion of Lactated Ringer's (Hospira, Lake Forest, IL, USA) to maintain hydration. A midline incision was made from the supraorbital process to the nuchal crest and a 14-mmdiameter circular craniotomy was trephined along the sagittal suture, positioning the center of the craniotomy $15 \mathrm{~mm}$ anterior to lambda, which is on the nuchal crest, and leaving the dura intact. A stainless steal custom threaded hub (Custom Design and Fabrication, Richmond, VA, USA) was screwed into the craniotomy site to a depth of $\sim 4 \mathrm{~mm}$. Screws were then placed directly posterior and anterior-lateral to the craniotomy, and dental acrylic (methyl-methacrylate; Hygenic Corp., Akron, OH, USA) was applied around the hub and screws to insure hub stability. The procedures used to induce cFPI in the micro pig were consistent with those described previously in the rodent [55]. This injury is induced by releasing a pendulum to impact a fluid-filled cylinder that generates a fluid pulse. The fluid pressure wave is transduced through the injury hub to the surface of the dura and ultimately to the cerebral spinal fluid and brain. Briefly, anesthetized micro pigs were connected to a central fluid percussion device retrofitted with a L-shaped stainless steal adaptor that allowed for a sealed connection to the injury hub. Micro pigs were then injured at a magnitude of $1.68 \pm 0.4$ atm with a pressure pulse measured by a transducer affixed to the injury device and displayed on an oscilloscope (Tektronix, Beaverton, OR, USA). Immediately after injury induction, animals were disconnected from the injury device, the screws and hub were removed from the bone, and the dental acrylic, hub, and screws were removed en bloc. This injury did not result in any breach of the dura mater. Gel foam was placed over the craniotomy/injury site, to alleviate minute bone bleeding, and the scalp was sutured. Animals were maintained under anesthetic for the duration of the 6-h postinjury monitoring period. Identical surgical procedures were followed for sham-injured animals, without the release of the pendulum to induce the injury.

\section{Physiological assessment}

To preclude the possibility that observed pathology was associated with TBI-induced systemic abnormalities, detailed physiological assessments were performed throughout the 6-h post-injury monitoring period. Heart rate, arterial blood pressure, rectal temperature, and hemoglobin oxygen saturation were monitored and recorded throughout the experiment via a $\mathrm{Cardell}^{\circ} \mathrm{MAX}-$ 12HD (Sharn Veterinary, Inc., Chicago, IL, USA). The femoral artery was cannulated for continuous monitoring of MABP and for blood sampling to determine arterial oxygen tension $\left(\mathrm{PaO}_{2}\right)$, arterial carbon dioxide pressure $\left(\mathrm{PaCO}_{2}\right)$, and $\mathrm{pH}$ values using a Stat Profile pHOx (NOVA Biomedical, Waltham, MA, USA). The resting $\mathrm{PaCO}_{2}$ level was maintained between 35 and $40 \mathrm{mmHg}$ by adjusting the rate and/or tidal volume of the respirator. All animals maintained physiological homeostasis (i.e., $60 \mathrm{mmHg}<\mathrm{MABP}<90 \mathrm{mmHg}$, hemoglobin oxygen saturation $>90 \%, 90 \mathrm{BPM}<$ heart rate < 140 BPM; Table 1).

\section{Tissue processing}

At 6-h post-sham or cFPI, micro pigs were overdosed with 3-ml euthasol euthanasia-III solution (Henry Schein, Dublin, OH, USA) transcardially perfused with $0.9 \%$ saline followed by $4 \%$ paraformaldehyde $/ 0.2 \%$ glutaraldehyde in Millonig's buffer (136 mM sodium phosphate monobasic/109 $\mathrm{mM}$ sodium hydroxide) for immunohistochemical analysis. After transcardial perfusion, the brains were removed and post-fixed in $4 \%$ paraformaldehyde/ $0.2 \%$ glutaraldehyde/Millonig's buffer for 36-48 h. Post-fixed brains were blocked into 5-mm coronal segments throughout the rostral-caudal extent using a tissue slicer (Zivic Instruments, Pittsburgh, PA, USA). In our hands, cFPI in the micro pig produced symmetrical bilateral microscopic pathology, which involved multiple brain loci. The burden of DAI, however,

Table 1 Systemic physiology was within normal ranges throughout the 6-h post-injury monitoring period

\begin{tabular}{llll}
\hline Variable & Group & Pre-injury & Post-injury \\
\hline Weight & Sham & $19.13 \pm 4.72$ & \\
& TBI & $20.12 \pm 3.37$ & \\
$\mathrm{pH}$ & Sham & $7.47 \pm 0.03$ & $7.48 \pm 0.03$ \\
& TBI & $7.49 \pm 0.03$ & $7.52 \pm 0.02^{*}$ \\
$\mathrm{paCO}_{2} \mathrm{mmHg}$ & Sham & $39.23 \pm 4.20$ & $37.92 \pm 1.37$ \\
& TBl & $40.83 \pm 2.81$ & $37.61 \pm 1.11$ \\
$\mathrm{paO}_{2} \mathrm{mmHg}$ & Sham & $585.25 \pm 53.92$ & $359.74 \pm 187.80$ \\
& TBl & $558.50 \pm 41.42$ & $492.18 \pm 116.09$ \\
$\mathrm{MABP} \mathrm{mmHg}$ & Sham & $94.29 \pm 14.94$ & $86.13 \pm 20.19$ \\
& TBI & $89.53 \pm 8.83$ & $79.90 \pm 8.24$ \\
$\mathrm{Hemoglobin}_{2}$ (\%) & Sham & $99.90 \pm 1.74 \times 10^{-14}$ & $99.19 \pm 0.61$ \\
& TBl & $99.83 \pm 0.167$ & $99.50 \pm 0.58$ \\
\hline
\end{tabular}

$T B I$ traumatic brain injury, MABP mean arterial blood pressure * Significant difference compared with sham values at same measurement point $p<0.05$. Values are mean \pm standard deviation of the mean 
was particularly consistent within the thalamus, leading us to focus on this region for the current communication. Segments containing the thalamus were bisected at the midline and the left side was analyzed. The 5-mm coronal segments containing the thalamus were coronally sectioned in $0.1 \mathrm{M}$ phosphate buffer with a vibratome (Leica, Bannockburn, IL, USA) at a thickness of $40 \mu \mathrm{m}$. Sections were collected serially in six-well plates ( $240 \mu \mathrm{m}$ between sections in each well) and stored in Millonig's buffer at $4{ }^{\circ} \mathrm{C}$. For the quantification of both axonal injury and microglial activation, a random well (1-6) was selected using a random number generator and six sections representing the rostral-caudal axis contained within the selected well were analyzed. All histological analyses were restricted to the thalamus using anatomical landmarks and were performed by an investigator blinded to animal injury (sham or mTBI).

\section{Detection and assessment of axonal injury}

To visualize the breakdown of axonal transport within the axonal segment proximal to its neuronal soma, a process indicative of DAI, immunofluorescence targeting the normally expressed and anterogradely transported amyloid precursor protein (APP) was performed.

For the preliminary assessment of DAI in the micro pig brain following cFPI sections from various brain regions throughout the rostra-caudal extent were blocked and permeabilized in $1.5 \%$ Triton/10 \% NGS/PBS followed by overnight incubation with the primary rabbit antibody against the C-terminus of $\beta$-APP (1:700; Cat.\# 51-2700, Life Technologies, Carlsbad, CA, USA) in $10 \%$ NGS/PBS at $4{ }^{\circ} \mathrm{C}$. A biotinylated goat anti-rabbit IgG (1:1000; Cat.\# BA-1000, Vector Laboratories, Burlingame, CA, USA) secondary antibody was used. The sections were then incubated in avidin biotinylated enzyme complex using the Vectastain ABC kit (Vector Laboratories, Burlingame, CA, USA) followed by visualization with $0.05 \%$ diaminobenzidine $/ 0.01 \% \mathrm{H}_{2} \mathrm{O}_{2} / 0.3 \%$ imidazole/PBS. The tissue was mounted, dehydrated, and cover-slipped. Visualization of APP-labeled axonal swellings was performed using a Nikon Eclipse 800 microscope (Nikon, Tokyo, Japan) equipped with an Olympus DP71 camera (Olympus, Center Valley, PA, USA).

To quantify the degree of thalamic DAI, immunofluorescence was used, in order to differentiate between the axonal swellings that contain a large amount of APP and the neuronal soma that contain lower amounts of APP. Briefly, six thalamic sections per animal (as detailed above) were blocked and permeabilized in $10 \%$ normal goat serum and $1.5 \%$ Triton followed by overnight incubation with a primary rabbit antibody against the C-terminus of $\beta$-APP (1:700; Cat.\# 51-2700, Life Technologies, Carlsbad, CA, USA) at $4{ }^{\circ} \mathrm{C}$. Secondary antibody, Alexa Fluor 568-conjugated goat anti-rabbit
IgG (1:500; Cat.\# A-11011, Life Technologies, Carlsbad, CA, USA) was then incubated and the tissue was mounted using Vectashield hardset mounting medium with Dapi (Cat.\# H-1500; Vector Laboratories, Burlingame, CA, USA). Tissue from all animals was processed concomitantly to obviate variability in staining intensity. Visualization of APP-labeled axonal swellings was performed using a Nikon Eclipse 800 microscope (Nikon, Tokyo, Japan) equipped with an Olympus DP71 camera (Olympus, Center Valley, PA, USA). Image acquisition settings were held constant for all animals. Images (60 images per animal; 10 images in each of the 6 sections assessed) were taken by a blinded investigator at $10 \times$ magnification $\left(0.72-\mathrm{mm}^{2}\right.$ field) in a systematically random fashion starting at the dorsal lateral aspect of the thalamus. Dapi signal was used for field advancement and to verify focus as well as restriction within the thalamus. A fluorescent intensity threshold was set for all images to eliminate any neuronal somatic expression of APP from the assessment. The number of $\mathrm{APP}^{+}$axonal swellings was analyzed using the particle analysis function in ImageJ software (NIH, Bethesda, MD, USA). The number of $\mathrm{APP}^{+}$swellings per unit area was quantified for each image and averaged for each animal.

\section{Detection and semi-quantification of microglia activation}

To identify microglia, immunohistochemistry against the calcium binding protein, Iba-1 (1:1000; Cat.\# 51-2700, Life Technologies, Carlsbad, CA, USA), was done. Briefly, six sections per animal (as explicated above) were blocked and permeabilized in $1.5 \%$ Triton/10 \% NGS/PBS followed by overnight incubation with the primary antibody in $10 \% \mathrm{NGS} / \mathrm{PBS}$ at $4{ }^{\circ} \mathrm{C}$. A biotinylated goat anti-rabbit IgG (1:1000; Cat.\# BA-1000, Vector Laboratories, Burlingame, CA, USA) secondary antibody was used. The sections were then incubated in avidin biotinylated enzyme complex using the Vectastain $A B C$ kit (Vector Laboratories, Burlingame, CA, USA) followed by visualization with $0.05 \%$ diaminobenzidine/ $0.01 \% \mathrm{H}_{2} \mathrm{O}_{2} / 0.3 \%$ imidazole/PBS. The tissue was mounted, dehydrated, and cover-slipped. Tissue from all animals was processed concomitantly to reduce variability between animals. The diffuse nature of the microscopic pathology, as well as the extensive area of the micro pig thalamus, precluded the use of traditional stereological quantification. Therefore, the entire thalamus was assessed for each of the six sections selected for each animal. Visualization of Iba-1-labeled microglia was performed using a Nikon Eclipse 800 microscope (Nikon, Tokyo, Japan). Identification of microglia activation was based on specific morphological criteria. Microglia with highly ramified fine process networks that were lightly labeled with Iba-1 were considered non-reactive, while microglia with thicker, shorter, or absent processes 
and darker Iba-1 labeling were identified as active/reactive [25-27]. The degree of microglia activation was assessed using a graded scale from 0 to $5 \quad(0=$ no microglial activation observed, $1=$ ramified microglia with thicker processes and darker Iba-1 labeling observed in $\sim 5 \%$ of the thalamus, $2=$ activated microglia observed in $\sim 5-10 \%$ of the thalamus, $3=$ activated microglia observed in $\sim 10<25 \%$ of the thalamus, $4=$ activated microglia observed in $\sim 25<$ $50 \%$ of the thalamus, and $5=$ activated microglia observed in $>50 \%$ of the thalamus). Two blinded investigators analyzed all sections independently and their scores were averaged for each animal.

\section{Tissue processing for microglia morphology and process contact analyses}

A subset of tissue sections taken from 9 injured and 3 sham animals were triple labeled with the following antibodies: rabbit anti $\beta$-APP (1:700; Cat.\# 51-2700, Life Technologies, Carlsbad, CA, USA), rabbit anti Iba-1 (1:1000; Cat.\# 019-19741, Wako, Osaka, Japan), and rat anti-myelin basic protein (1:1000; Cat\#NB600-717, Novus Biologicals, Littleton, CO, USA). To reduce the amount of lipid within the tissue and enhance antibody penetration, the sections were dehydrated then rehydrated through varying percentages of ethanol, with the tissue beginning and ending in PBS. The tissue was blocked and permeabilized with $10 \%$ NGS/1.5 \% Triton/PBS followed by incubation with the rabbit anti-APP antibody over night at $4{ }^{\circ} \mathrm{C}$ followed by incubation with Alexa Fluor 488-conjugated goat anti-rabbit secondary antibody (1:500; Cat.\# A11034, Life Technologies, Carlsbad, CA, USA). Tyramide amplification of the APP signal (primary antibody diluted to 1:5000) was performed on the majority of sections to further reduce the risk of crossreaction with the Iba- 1 antibody, using a rabbit Alexa- 488 conjugated Tyramide amplification kit (Cat.\# T20922, Life Technologies, Carlsbad, CA, USA) according to the manufacturer's instructions. Following APP immunolabeling, the tissue was blocked once again with $10 \%$ NGS then incubated with the rabbit anti-Iba-1 and rat anti-MBP antibodies. Alexa Fluor 568-conjugated goat anti-rat IgG (1:500; Cat.\# A11077, Life Technologies, Carlsbad, CA, USA) and Alexa Fluor 633-conjugated goat anti-rabbit IgG (1:500; Cat.\# A21071, Life Technologies, Carlsbad, CA, USA) were used for the visualization of MBP and Iba-1, respectively. Controls to assess cross-reactivity among all antibodies were performed using the following antibody combinations: no primary/rabbit Alexa-488 (or rabbit Alexa-488 tyramide amplification), rat Alexa-568, rabbit Alexa-633; rat anti-MBP primary/rabbit Alexa-633 secondary; rabbit anti-Iba-1 primary/rat Alexa-568 secondary; rabbit anti-APP primary (either 1:700 or 1:5000 followed by second blocking step/rabbit Alexa-633 secondary). For all controls, any cross-reactivity was below background detection limits (data not shown). Tissue was mounted using Vectashield hardset mounting medium with Dapi (Cat.\# H1500; Vector Laboratories, Burlingame, CA, USA) and $z$-stacked images $(10-25$ - $\mu \mathrm{m}$-thick stacks; $0.32 \mu \mathrm{m}$ between steps; sham $=6 z$-stacks, injured $=19 z$-stacks) were captured on a Zeiss LSM 710 confocal microscope (Carol Zeiss, Oberkochen, Germany). Regions for imaging were selected based on the APP profile for injured animals and the penetration of MBP for the sham animals. Three-dimensional reconstructions of the $z$-stacks were made using Velocity software (PerkinElmer, Waltham, MA, USA).

\section{Microglia morphology analysis}

For the morphological analysis of microglia, 2D representations of the Iba-1-labeled Alexa 633 channel 3D reconstructions (sham $=1$ reconstruction from each of 3 animals; injured $=1$ reconstruction from each of 9 animals) were used. Each individual cell with an identifiable soma and process network within the reconstruction was assessed. The soma size, the total number of processes (with each new branch point designating the origin of a new process), the average process length, the number of primary processes that directly originate from the soma, and the number of terminal process endpoints/tips for each microglial cell were quantified manually using Image J software $(\mathrm{NIH}$, Bethesda, MD, USA). These morphological features and particularly the assessment of total number of processes and the number of terminal process endpoints provide detailed data indicating the complexity/ramification of the process network of individual microglia.

\section{Microglia process contact analyses}

Triple-labeled 3D reconstructions performed using Velocity software were used for the analysis of microglia process contacts with DAI in injured micro pigs or intact axonal segments in sham animals. All APP+ axonal swellings contained within each 3D image were assessed in injured animals and 10 myelinated fibers per 3D image, chosen via random-number generator determined $x, y$ coordinates, were assessed for sham animals. The length of the proximal axonal swelling (from APP+ axonal stem to the disconnected base of APP+ swelling) or the intact myelinated MBP+ axonal segment for sham animals was measured using Velocity software. Microglia process contacts were identified manually on 3D images and confirmed by stepping through the $z$-stacks. All microglial contacts within the delineated axonal segments were included in the analysis. 


\section{Electron microscopy}

To evaluate the ultrastructural characteristics of DAI in the micro pig thalamus while verifying microglia process contacts on these axonal swellings, a subset of tissue was immunolabeled with either rabbit anti $\beta$-APP (1:700; Cat.\# 51-2700, Life Technologies, Carlsbad, CA, USA) or rabbit anti Iba-1 (1:1000; Cat.\# 019-19741, Wako, Osaka, Japan), followed by incubation with biotinylated goat anti-rabbit IgG (1:1000; Cat.\# BA-1000, Vector Laboratories, Burlingame, CA, USA) secondary antibody. The reaction product was visualized with $0.05 \%$ diaminobenzidine/0.01\% hydrogen peroxide/ $0.3 \%$ imidazole in $0.1 \mathrm{M}$ phosphate buffer and the tissue was prepared for EM analysis. In this approach, tissue sections were osmicated, dehydrated, and embedded in epoxy resin on plastic slides. After resin curing, the slides were studied with routine light microscopy to identify the precise thalamic areas for excision. Once identified, these sites were removed, mounted on plastic studs, and 70-nm sections were cut serially and mounted on Formvar-coated slotted grids. The grids were stained in $5 \%$ uranyl acetate in $50 \%$ methanol and $0.5 \%$ lead citrate. Ultrastructural qualitative analysis was performed using a JEOL JEM 1230 transmission electron microscope (JEOL-USA, Peabody, MA, USA) equipped with Ultrascan 4000SP CCD and Orius SC1000 CCD cameras (Gatan, Pleasanton, CA, USA).

\section{Statistical analysis}

Data was tested for normality using a Shapiro-Wilk analysis. Normally distributed data was analyzed via oneway analysis of variance (ANOVA). Non-parametric data were analyzed using a Mann-Whitney $U$ test. Statistical significance was set at a $p$ value $<0.05$. Data are presented as mean \pm standard error of the mean (SEM).

\section{Results}

Mild diffuse traumatic brain injury does not generate physiologic or macroscopic pathology in the pig

The cFPI used in this study has been successfully employed in rodents for decades to mimic the movement of the brain within the cranial vault following noncontusive diffuse mTBI with high efficacy and consistency [55]. This injury generated virtually no macroscopic pathology in the micro pig brain (Fig. 1). While limited subarachnoid bleeding, particularly overlying the occipital cortex and cerebellum, was observed, macroscopic hemorrhage within the brain parenchyma was not detected. Isolated petechial hemorrhage was observed in a few injured animals, however, this did not interfere with any of the analyses performed. Additionally, these injuries were not accompanied by contusion, hematoma formation, ventricular enlargement, or tissue loss throughout the rostral-caudal extent of the micro

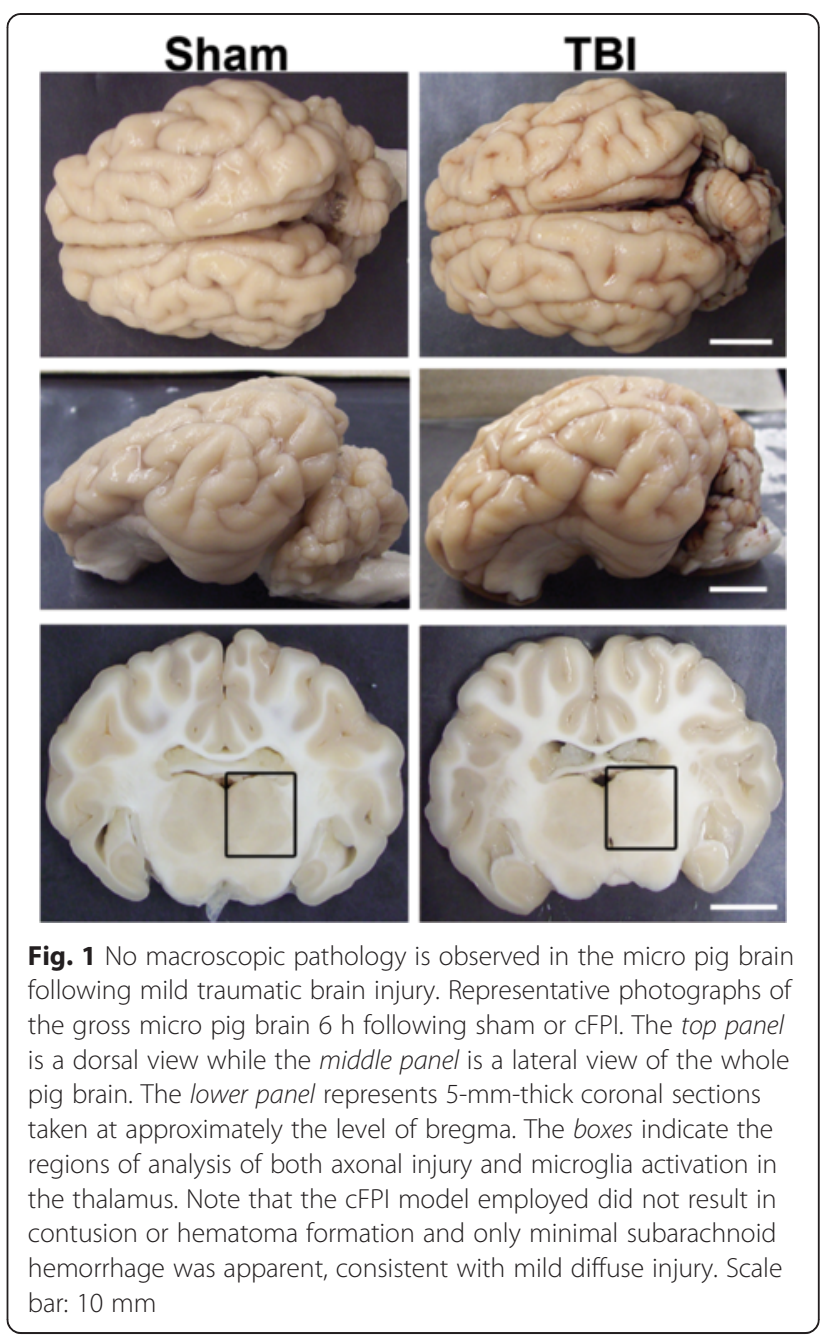

pig brain. Collectively, these features speak to the mild diffuse nature of the injury employed.

To evaluate the possibility of confounding systemically induced change in this model, each animal's systemic physiology was closely monitored for the duration of the experiment, both prior to the induction of injury and for the entire 6-h post-sham or injury period. Core temperature was also monitored and maintained at $37{ }^{\circ} \mathrm{C}$ to negate the possibility of protection due to hypothermia. As depicted in Table 1, averages of the pre- and postinjury physiologic values, taken throughout the 6-h postinjury monitoring period, were primarily consistent with sham-injured control animals (one-way ANOVA, weight $F_{1,19}=0.199, p=0.661$; pre-injury $\mathrm{pH} F_{1,19}=2.169, p=$ 0.157 ; pre-injury $\mathrm{paCO}_{2} F_{1,19}=0.732, p=0.403$; postinjury $\mathrm{paCO}_{2} F_{1,19}=0.199, p=0.660$; pre-injury $\mathrm{paO}_{2}$ $F_{1,19}=1.000, p=0.330$; post-injury $\mathrm{paO}_{2} F_{1,19}=2.860$, $p=0.107$; pre-MABP $F_{1,15}=0.150, \quad p=0.704$; postinjury MABP $\quad F_{1,15}=0.115, \quad p=0.739 ; \quad$ pre-injury hemoglobin $\mathrm{O}_{2} \quad F_{1,19}=0.435, \quad p=0.518$; post-injury hemoglobin $\mathrm{O}_{2} \quad F_{1,19}=0.739, p=0.401 ;$ sham $n=3$, 


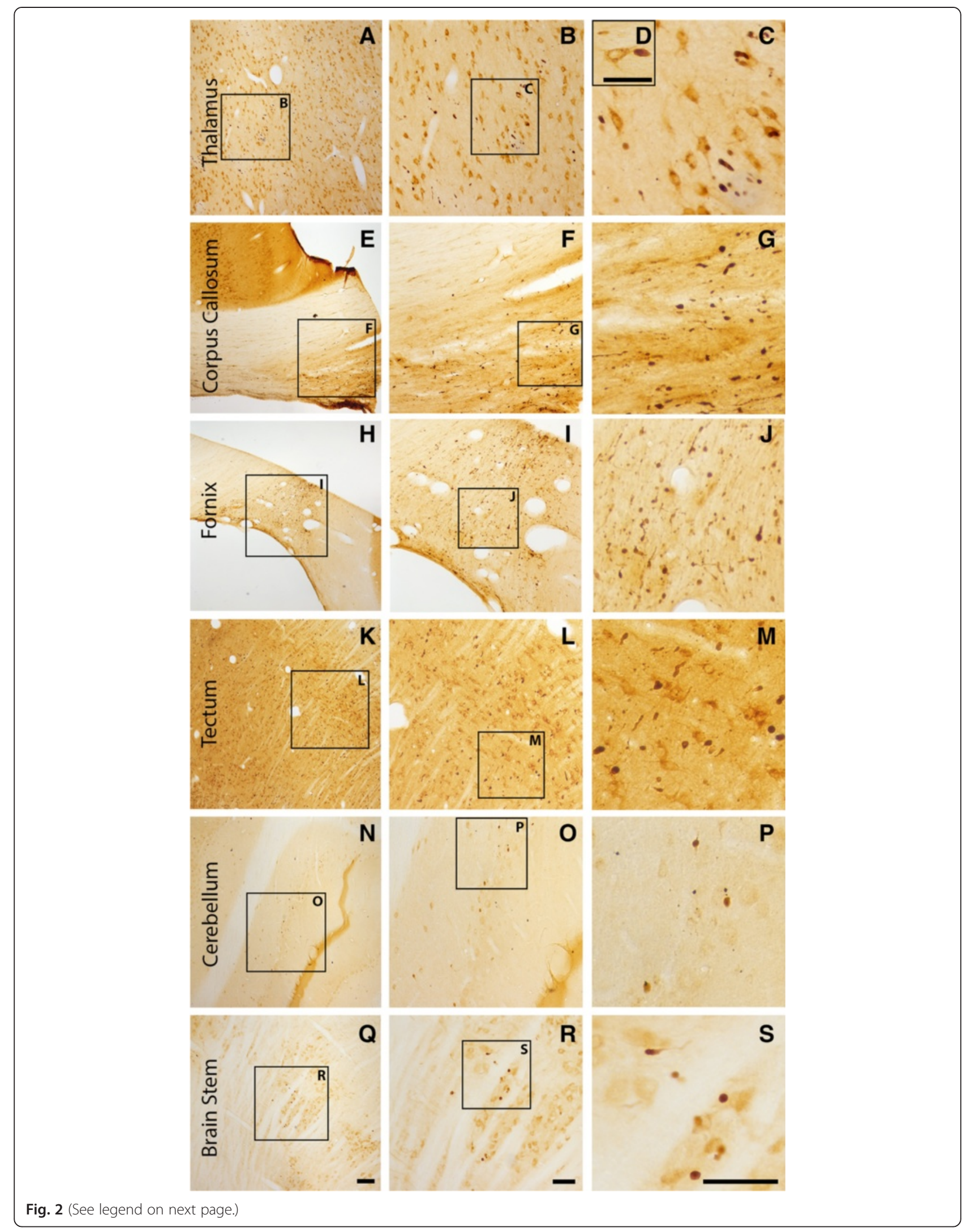


(See figure on previous page.)

Fig. 2 Axonal injury is observed in various regions throughout the micro pig brain following cFPI. Representative photomicrographs of APP immunohistochemistry in regions of the micro pig brain that demonstrated DAl in animals sustaining cFPI. Images in the middle panel (b, $\mathbf{f}$, i, I, o, $\mathbf{r}$ ) are magnified regions indicated in the images of the left panel $(\mathbf{a}, \mathbf{e}, \mathbf{h}, \mathbf{k}, \mathbf{n}, \mathbf{q})$ and images in the right panel (c, $\mathbf{g}, \mathbf{j}, \mathbf{m}, \mathbf{p}, \mathbf{s})$ are magnified regions indicated in the middle panel $(\mathbf{b}, \mathbf{f}, \mathbf{i}, \mathbf{l}, \mathbf{o}, \mathbf{r})$, respectively. Note that DAI within the thalamus and tectum was diffusely distributed throughout the domain, while DAl within the other regions was more localized. Also note that while not common, APP+ proximal axonal swellings in continuity with the neuronal soma (d) were observed in the thalamus. Scale bar in q: $200 \mu \mathrm{m} ; \mathbf{r}$ and $\mathbf{s}: 100 \mu \mathrm{m} ; \mathbf{d}: 50 \mu \mathrm{m}$

mTBI $n=18)$. Post-injury $\mathrm{pH}$, however, was slightly higher than sham $\left(F_{1,19}=6.174, p=0.022\right)$. Importantly, these values were all within normal ranges throughout the experiment (Table 1). The $\mathrm{paO}_{2}$, both prior to and following injury, was higher than typically reported due to our use of $100 \% \mathrm{O}_{2}$. While these values are higher than are typically observed, the $\mathrm{paO}_{2}$ remained well below the lower limit for oxygen toxicity $[56,57]$. Importantly, since all physiologic parameters evaluated were within normal ranges, pathology observed could be attributed to the mTBI and not to additional systemic physiological changes.

\section{Extensive DAI is apparent in the thalamus following mTBI} Since axonal injury is a pathological hallmark associated with much of the morbidity following TBI [10, 11, 14-18], the extent of DAI was qualitatively assessed in various regions of the micro pig brain $6 \mathrm{~h}$ following sham or cFPI. This time point was chosen, based on our initial observations in this model, which revealed robust DAI by 6-h post-injury in the micro pig brain (unpublished findings). Pronounced DAI, identified as APP+ proximal axonal swellings, indicative of impaired protein transport in the proximal axonal segment remaining attached to the neuronal soma following disconnection [53, 54, 58, 59], was observed in the thalamus, corpus callosum, fornix, tectum of the midbrain, cerebellum, and brainstem (Fig. 2). While, in some animals, other brain regions displayed more densely localized DAI, diffuse thalamic DAI was the most consistent finding across animals. As thalamic damage is also a common occurrence in human TBI [49-52], we concentrated our quantitative histological analysis on this anatomical region (one-way ANOVA $F_{1,19}=6.677, p=0.018$; sham $n=3$, TBI $n=18$; Fig. 3 ) Consistent with previous studies examining DAI via APP accumulation, no APP+ axonal swellings were observed in sham-injured micro pigs (Fig. 3a). Following cFPI, however, substantial APP+ proximal axonal swellings were apparent throughout the thalamic domain (Fig. 3). DAI within the micro pig thalamus appeared as large $(\sim 5 \mu \mathrm{m}$ in diameter $) \mathrm{APP}+$ spheroids diffusely distributed in patches throughout the dorsalventral and rostral-caudal extent of the thalamus.

To explore the subcellular pathology of DAI in the micro pig thalamus $6 \mathrm{~h}$ following diffuse mTBI, the ultrastructure of these APP+ proximal axonal swellings was assessed (Fig. 4). The majority of proximal axonal swellings, as identified by immunoelectron microscopy against APP, demonstrated ultrastructural alterations consistent with axonal damage. Disordered and clumped neurofilaments were commonly observed within APP containing axonal swellings, with some cases displaying a neurofilamentous core surrounded by organelles (Fig. 4c). Other proximal swellings exhibited predominant organelle accumulation. These findings were consistent with ultrastructural descriptions of human TBIinduced acute axonal pathology [60]. These subcellular changes in the proximal axonal swellings were not, however, consistent with the initiation of Wallerian degeneration [12, 58], which was observed in distal axonal swellings that lacked APP immunoreactivity
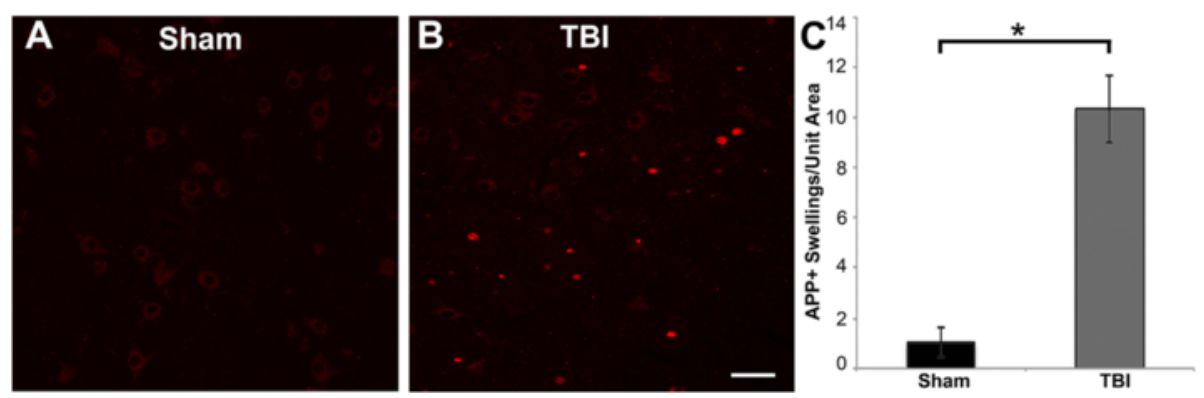

Fig. 3 Abundant DAI is readily apparent $6 \mathrm{~h}$ following cFPI in the micro pig thalamus. Representative photomicrographs of APP immunofluorescence in the thalamus of animals sustaining sham (a) or cFPI (b). While sham-injured animals had little to no APP labeling, prevalent APP+ axonal swellings, indicative of DAl, were apparent following injury. c Bar graph depicting the average number of APP labeled axonal swellings/ 0.72 mm ${ }^{2}$ of thalamic tissue. Graph depicts mean \pm standard error of the mean. ${ }^{*} p<0.05$. Scale bar: $50 \mu \mathrm{m}$ 


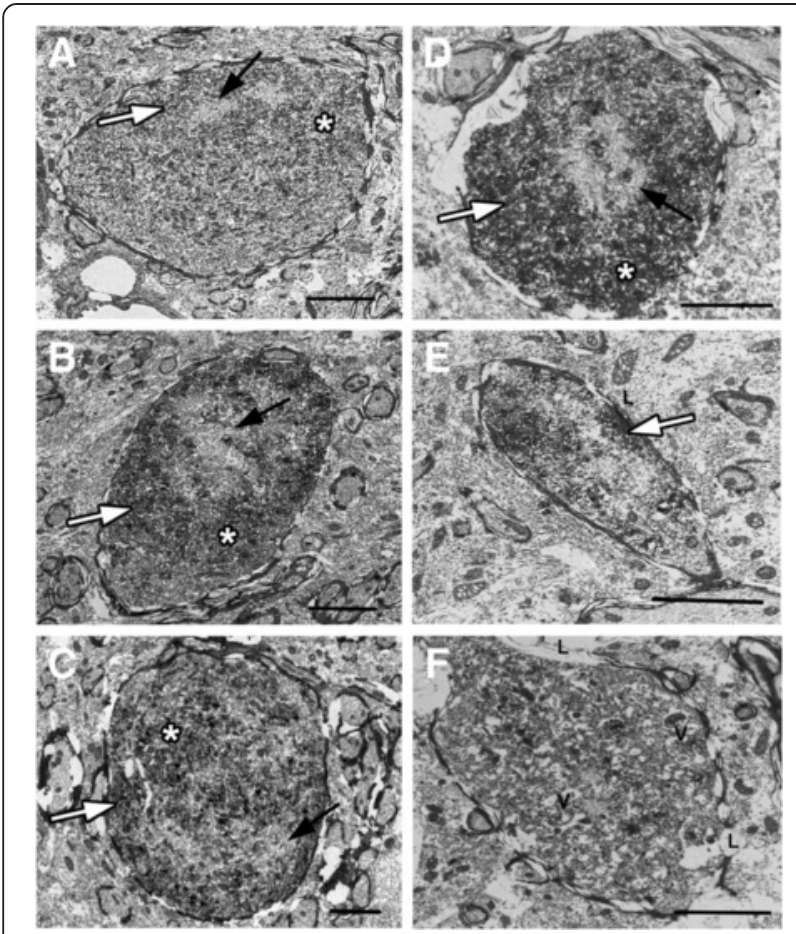

Fig. 4 Ultrastructural characteristics of acute DAI within the micro pig thalamus are consistent with human DAI. Representative electron micrographs of axonal swellings labeled with (a-e) or without (f) APP (white arrows). a-e Consistent with ultrastructural axonal pathology in humans, APP+ proximal axonal swellings in the pig following central fluid percussion injury display clumped disordered neurofilaments (black arrows) and areas of organelle accumulation (asterisks). f Distal axonal swellings, lacking APP labeling, demonstrate characteristics consistent with Wallerian degeneration, including, vacuolization ( $V$ ) and lucent zones surrounding aspects of the swelling indicating axolemmal or myelin disruption (L). Scale bar b-e: $2 \mu \mathrm{m}$

(Fig. 4f). These APP - distal axonal swellings displayed vacuolization and/or increase cytoplasmic electron density consistent with Wallerian change. Distal, APP-, axonal swellings also displayed areas of axolemmal or myelin disruption, reflected in lucent zones surrounding aspects of the swelling (Fig. 4f).

\section{Acute thalamic microglia activation following mTBI occurs in areas sustaining DAI}

Neuroinflammation, as identified by Iba- $1+$ microglia with activated morphologies, within the micro pig thalamus was assessed using a graded scale from 0 to $5(0=$ no observed microglial activation and $5=$ activated microglia observed in $>50 \%$ of the coronal thalamic section; sham $n=3$, TBI $n=18$ ). While the microglia within the sham thalami were evenly distributed and primarily non-reactive, with spindly ramified process networks that were lightly labeled with Iba-1, some isolated microglia demonstrated thicker, shorter processes with more substantial Iba-1 labeling (Fig. 5a-d). Following cFPI, however, microglia activation was pervasive, with pockets of morphologically active microglia, exhibiting heavy Iba-1 labeling, thicker processes, and less complex process networks, dispersed throughout the micro pig thalamus (Mann-Whitney $U$ test, $p=0.006$; Fig. 5e-h).

The process network of individual microglial cells was analyzed in either sham or injured thalami to verify the morphological alterations observed using the above semi-quantitative assessment (Mann-Whitney $U$ test: soma size $p=0.613$, process length $p=0.994$, total process number $p=0.001$, primary process number $p=$ 0.059 , process endpoints $p=0.001$; sham $n=30$ cells, TBI $n=70$ cells). The microglia in sham-injured micro pigs had ramified/complex process networks, reflected primarily by the total process number and the number of terminal process endpoints per cell (Fig. 5). Microglia within the thalamus of micro pigs sustaining mTBIinduced DAI, however, had fewer overall processes and less terminal process endpoints, indicative of a less complex and/or ramified process network (Fig. 5i, l, n). While the soma size, number of primary processes, and the average process length remained comparable between sham microglia and microglia following mTBI, the drastic reduction in the complexity/ramification of the microglial process network with injury confirms morphological alterations consistent with resident microglial activation [25-27, 61, 62] .

Interestingly, the degree of microglial activation within the thalamus, as assessed via the microglial activation score, was significantly correlated to the amount of DAI observed in each animal (Spearman's rho correlation coefficient $=0.763, \quad p=0.000 ; n=21$ ). Additionally, the areas of dense microglial activation appeared consistent with the thalamic sites that demonstrated the highest degree of DAI. Therefore, the spatial relationship between activated Iba-1+ microglia and DAI within the injured thalamus was assessed. As depicted in Fig. 6, regions that contained DAI also contained a plethora of morphologically activated microglia, whereas, areas in the injured thalamus that did not sustain DAI primarily contained non-reactive, ramified microglia.

\section{Microglia processes preferentially contact DAI proximal axonal swellings acutely post-injury}

To investigate the possibility that activated microglial processes converge on proximal axonal swellings sustaining mTBI-induced DAI in the micro pig thalamus, the number of microglia processes that contacted APP+ axonal swellings in injured animals or normal myelinated axons in sham animals was assessed (Mann-Whitney $U$ test $p=0.018$; sham $n=60$ axonal segments, TBI $n=60$ axonal swellings). Following sham injury, microglia processes made contact with myelinated axons, however, these contacts were sparse (Fig. 7a) and 

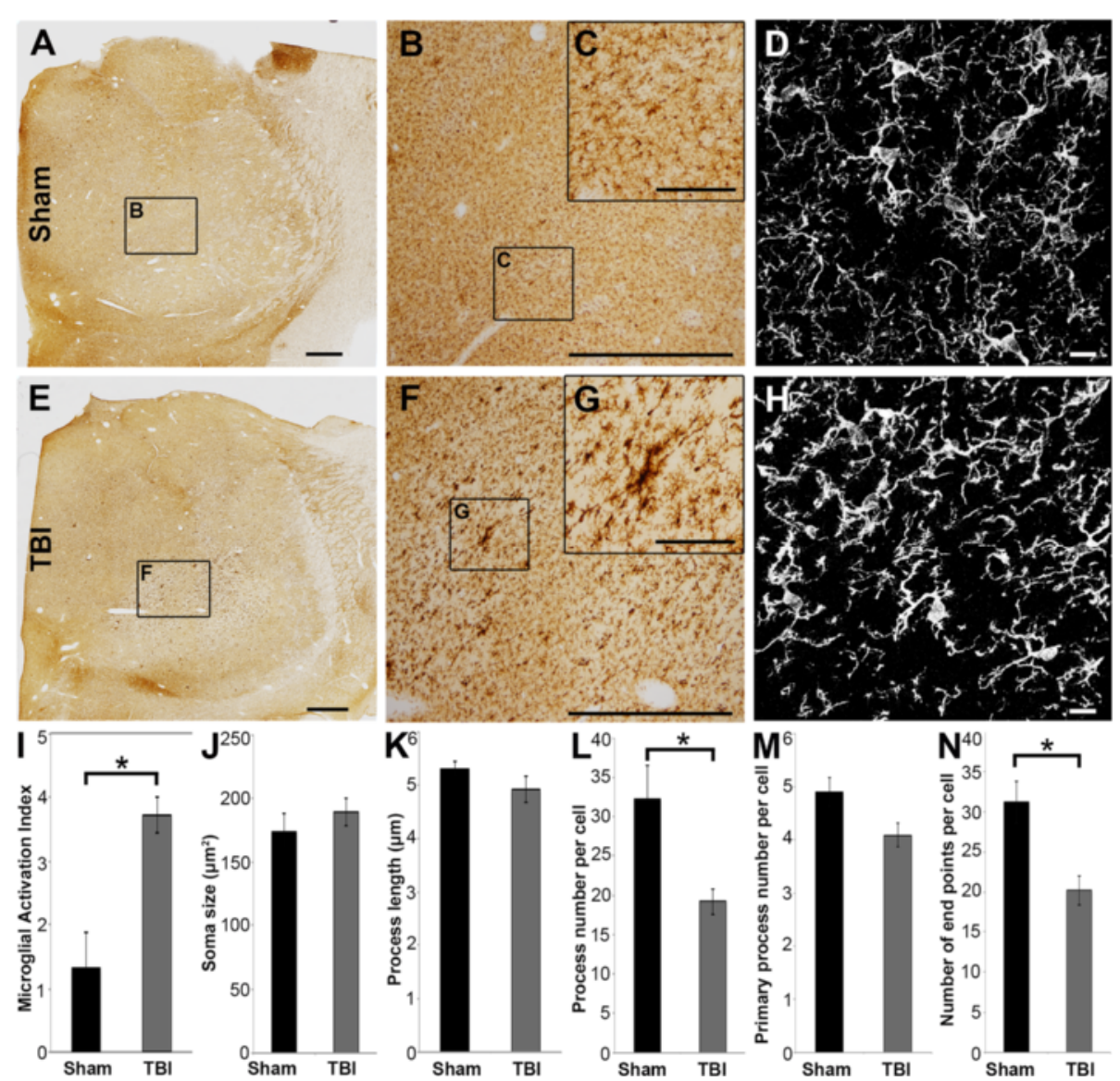

Fig. 5 Extensive microglial activation is observed in the micro pig thalamus $6 \mathrm{~h}$ following diffuse mTBI. Representative photomicrographs of the microglial marker lba-1 in the thalamus of sham-injured (a-d) or central fluid percussion injured (e-h) micro pigs. $\mathbf{b}$ and $\mathbf{f}$ are magnified regions indicated in $\mathbf{a}$ and $\mathbf{e}$, and $\mathbf{c}$ and $\mathbf{d}$ are magnified regions indicated in $\mathbf{b}$ and $\mathbf{f}$, respectively. $\mathbf{d}$ and $\mathbf{h}$ are two-dimensional flattened images of three-dimensional stacks through microglia in the sham (d) or injured thalamus (h). Note that the microglia appear ramified in sham-injured animals, indicating a quiescent state. While ramified microglia are present in brain-injured pigs, a large proportion of microglia have retracted, amoeboid or stellate morphologies, indicating activation. Bar graphs illustrating the degree of microglial activation in the thalamus (i) as well as the average soma size $(\mathbf{j})$, average process length $(\mathbf{k})$, average process number $(\mathbf{I})$, average number of primary processes $(\mathbf{m})$, and average number of end points ( $\mathbf{n}$ ) per Iba-1+ cell. Scale bar $\mathbf{a}$ and $\mathbf{e}=1 \mathrm{~mm}, \mathbf{b}$ and $\mathbf{f}=200 \mu \mathrm{m}, \mathbf{c}$ and $\mathbf{g}=40 \mu \mathrm{m}$, $\mathbf{d}$ and $\mathbf{h}=10 \mu \mathrm{m}$. Graph depicts the mean \pm standard error of the mean. ${ }^{*} p<0.05$

primarily consisted of microglia processes passing by and/or crossing over myelinated axons (sham $=63.39 \%$ of total contacts, $\mathrm{TBI}=25.23 \%$ of total contacts). This is shown in more detail in an additional movie file (Additional file 1). Conversely, nearly double the number of microglia processes contacted APP+ proximal axonal swellings following diffuse mTBI (Fig. 7). The majority of these contacts were bulbous end processes $(\mathrm{TBI}=$ $58.16 \%$ of total contacts, sham $=36.61 \%$ of total contacts) as apposed to processes passing over axons, which were common in the sham. An additional movie file represents these finding in more detail (Additional file 2). The percentage of microglial processes that appeared to cradle or "cup" the axon were also increased when contacting APP+ swellings compared to sham myelinated axons $(\mathrm{TBI}=16.60 \%$ of total contacts, sham $=6.09 \%$ of total contacts).

To further evaluate the increase in microglial process convergence on proximal segments of axons sustaining DAI, qualitative ultrastructural analysis of microglia morphology and process contacts were performed utilizing immunoelectron microscopy targeting Iba-1 (Fig. 8). Microglial cell bodies were easily recognized in sham thalami, however microglial processes were fine and widely dispersed with few processes directly associating with axons (Fig. 8a, b). Following diffuse mTBI, both the Iba-1+ microglial processes and soma were more prominent. As previously described, microglia with myelin and other cellular debris, indicative of active phagocytosis, were present in areas of Wallerian degeneration 


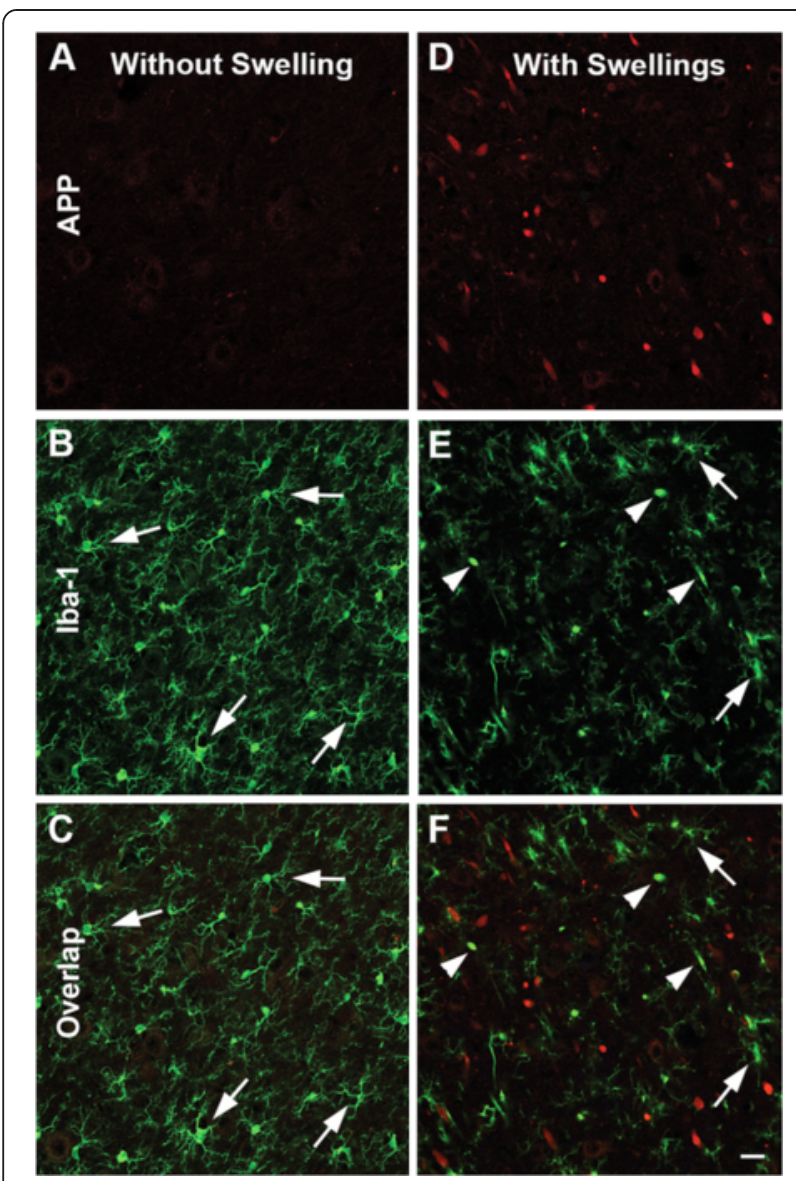

Fig. 6 Microglia activation occurs in thalamic sectors sustaining acute DAl 6 h following mTBI. Representative confocal micrographs of APP (red; $\mathbf{a}$ and $\mathbf{d}$ ) and Iba-1 (green; $\mathbf{b}$ and $\mathbf{e}$ ), with overlays in $\mathbf{c}$ and $\mathbf{f}$, in the thalamus of the same injured animal. Interestingly, areas lacking axonal injury (a-c) appear to contain inactive ramified microglia (arrows), whereas thalamic sites exhibiting DAl, indicated by accumulation of APP in axonal swellings $(\mathbf{d}-\mathbf{f})$, also appear to contain the majority of morphologically activated Iba-1+ microglia (arrow heads). Scale bar: $20 \mu \mathrm{m}$

(Fig. 8c). In the presence of proximal axonal swellings, as ultrastructurally identified using the common features observed and displayed in Fig. 4, however, microglia did not display sub-cellular alterations consistent with phagocytosis. Rather, small Iba-1+ points of contact were observed (Fig. 8d, e). In some cases, the microglial processes could be traced from the site of axonal contact back to the microglia soma, however, the majority of processes passed out of the plane of section (Fig. 8e, f). These data suggest that while microglia do phagocytize debris from the degenerating distal axon, they do not participate in phagocytosis of the proximal axonal swelling acutely post-mTBI, rather microglial processes appear to preferentially contact and/or converge on proximal axonal swellings.

\section{Discussion}

The current study demonstrates acute microglial process convergence on proximal swellings of axons sustaining acute DAI in the micro pig thalamus, an association not previously recognized in the literature. Throughout this study, we employed a cFPI model in adult micro pigs to evaluate the diffuse pathology associated with mTBI in a higher order gyrencephalic animal more comparable to humans $[47,48]$. This cFPI model, which has been successfully employed in rodents for decades, involves the transmission of a fluid pressure pulse to the brain and CSF through the intact dura mater, mimicking the movement of the brain following non-contusive diffuse mTBI in a reproducible fashion [55, 58, 63]. Using this model of cFPI, we demonstrated that diffuse mTBI in the micro pig produced substantial DAI, particularly within the thalamus, an area commonly affected in human TBI [49-52], without concomitant contusion or hematoma formation. Importantly, micro pig DAI was shown to be ultrastructurally consistent with that seen in humans [60] indicating that this model replicates human-like features of mTBI-associated diffuse pathology.

In concert with this axonal damage, extensive neuroinflammation, as identified by morphological alterations, indicative of microglial activation [25-27], was observed in the thalamus of micro pigs acutely following mTBI. Neuroinflammation is common following CNS injury in a variety of disease states and has been observed years following TBI in the human population [37, 51, 62, 64-69]. This chronic neuroinflammatory response has recently been observed in rodent models of trauma as well and is thought to be associated with negative outcomes both clinically and experimentally [19-23]. For a decade, it has been accepted that microglia react to CNS injury within minutes [70], however, little is known regarding acute neuroinflammation occurring minutes to hours following diffuse mTBI.

Previous studies, including our own, have focused on the phagocytic activity of microglia sub-acutely (days) following injury in rodents [29, 35-37, 39]. Specifically, we and others have identified an association between microglia phagocytosis and injured axons undergoing Wallerian degeneration days following injury in the rodent. Sub-acute phagocytosis has also been observed following demyelination and axonal injury in models of multiple sclerosis and leukodystrophy as well as during synaptic pruning during normal development [30, 62, $65,71,72]$. It is possible, due to the fact that the current study only assess microglia at one acute time-point post$\mathrm{mTBI}$, that the observed process convergence on proximal axonal swellings is an early indication of microglial progression to phagocytic reactivity. Microglia, however, have non-phagocytic functions that are less well understood [28-33]. In healthy brain, endogenous microglia 

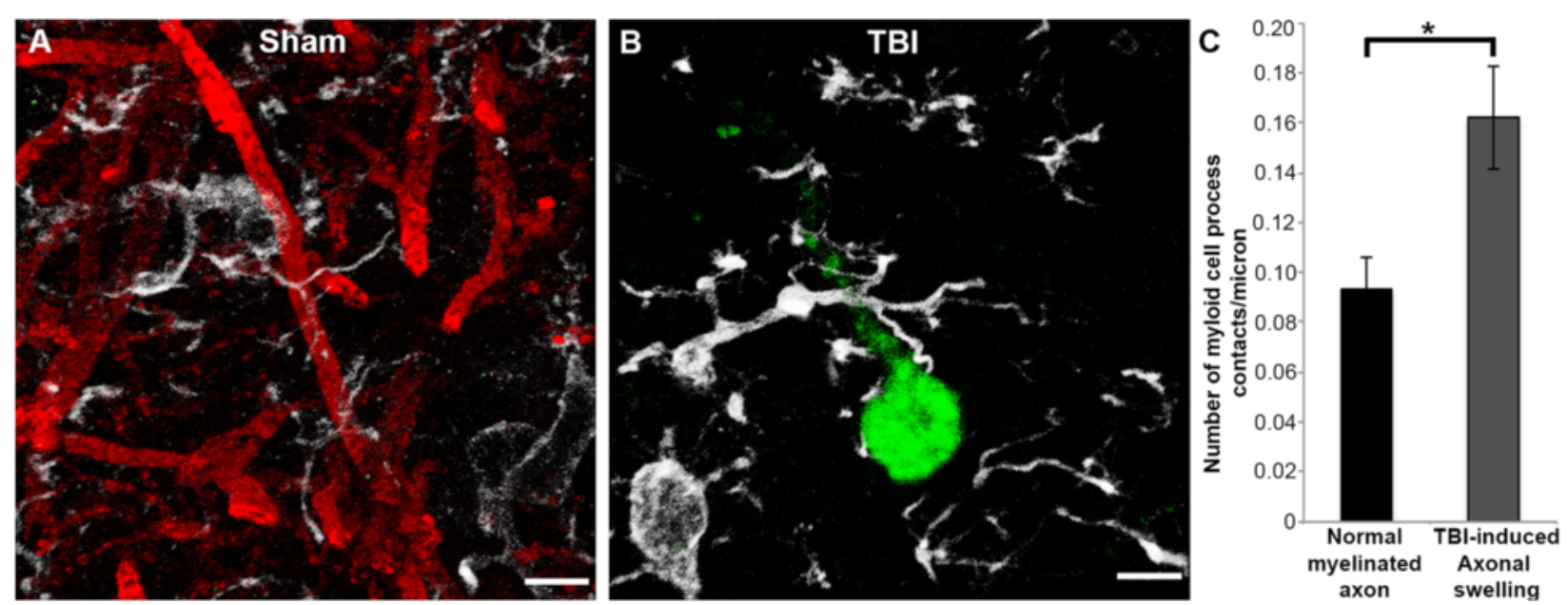

Fig. 7 Microglia processes appear to preferentially contact TBI-induced proximal axonal swellings. Representative 3D reconstructions of MBP+ myelinated axons (red) or APP+ axonal swellings (green) and Iba-1+ microglia (white) in sham-injured (a) or central fluid percussion injured (b) thalami. c Bar graph depicting the average number of Iba-1+ microglial processes contacting either MBP+ myelinated fibers in the sham animals or APP+ axonal swellings in injured animals. Graph depicts the mean \pm standard error of the mean. ${ }^{*} p<0.05$. Scale bar: $5 \mu \mathrm{m}$

have been shown to survey the parenchyma by extending highly dynamic processes that interact with the other cells of the CNS [73, 74]. This dynamic microglial process remodeling is altered under the influence of neuronal signals [27, 70, 75-77]. Additionally, a low calcium environment can activate microglial process convergence on neurons [78]. Microglial processes have also recently been shown to preferentially contact and functionally alter neurons with specific electrophysiological characteristics [61, 79].

While our study did not explore dynamic process remodeling, rather it assessed a snapshot of process dynamics acutely following mTBI, our findings indicate that non-phagocytic microglia processes preferentially interact with and/or converge on the proximal aspects of diffusely injured axons. Our previous studies and clinical observation indicate that the proximal axonal segments of neurons sustaining DAI do not immediately progress to cell death. Rather, they plastically adapt and remodel [14, 80-82], precluding the likelihood of acute phagocytosis. Calcium influx, however, has been well documented to occur acutely in axons sustaining DAI $[83,84]$. This influx might alter the calcium concentration in the extracellular space immediately surrounding the proximal axonal swelling, which could act as a signal for microglial process convergence. Additionally, diffusely injured axons display acute electrophysiological alterations [85-87] which could modulate microglial responses.

Activated microglia are typically categorized into different subgroups of M1, inflammatory microglia, or alternatively activated/anti-inflammatory M2 microglia. These sub-groups, which have been observed in rodents, humans, and pigs, express either pro- (M1) or anti(M2) inflammatory cytokines and are activated via different signaling pathways [29, 47, 88-91]. The specific functions of M2-activated microglia following mTBI are not well understood, however, it is speculated that M2 microglia are neuroprotective [22, 92, 93]. It is possible that the M2-activated microglia population is preferentially associated with the proximal swellings undergoing DAI, however, due to the limited study of neuroinflammation in pig, further investigation would be required to verify this potential phenomenon.

\section{Conclusions}

In summary, we demonstrated that central fluid percussion injury in adult micro pigs precipitated substantial acute axonal injury in the thalamus that is ultrastructurally similar to acute DAI in humans. Extensive microglial activation was also observed in the same thalamic sites, which directly correlated to the burden of thalamic axonal injury. The physical relationship between activated microglial processes and the proximal swelling of axons sustaining acute DAI was dramatically increased compared to uninjured myelinated thalamic axons in sham animals. In aggregate, these studies reveal acute microglial process convergence on proximal axonal swellings undergoing DAI, an interaction not previously recognized in the literature.

Current clinical techniques have emerged that allow for the visualization of neuroinflammation in the human population via PET imaging [51, 64, 94]. Our current finding that early microglial activation is directly associated with acute DAI in the micro pig, paired with the similarities between pig and human inflammatory 


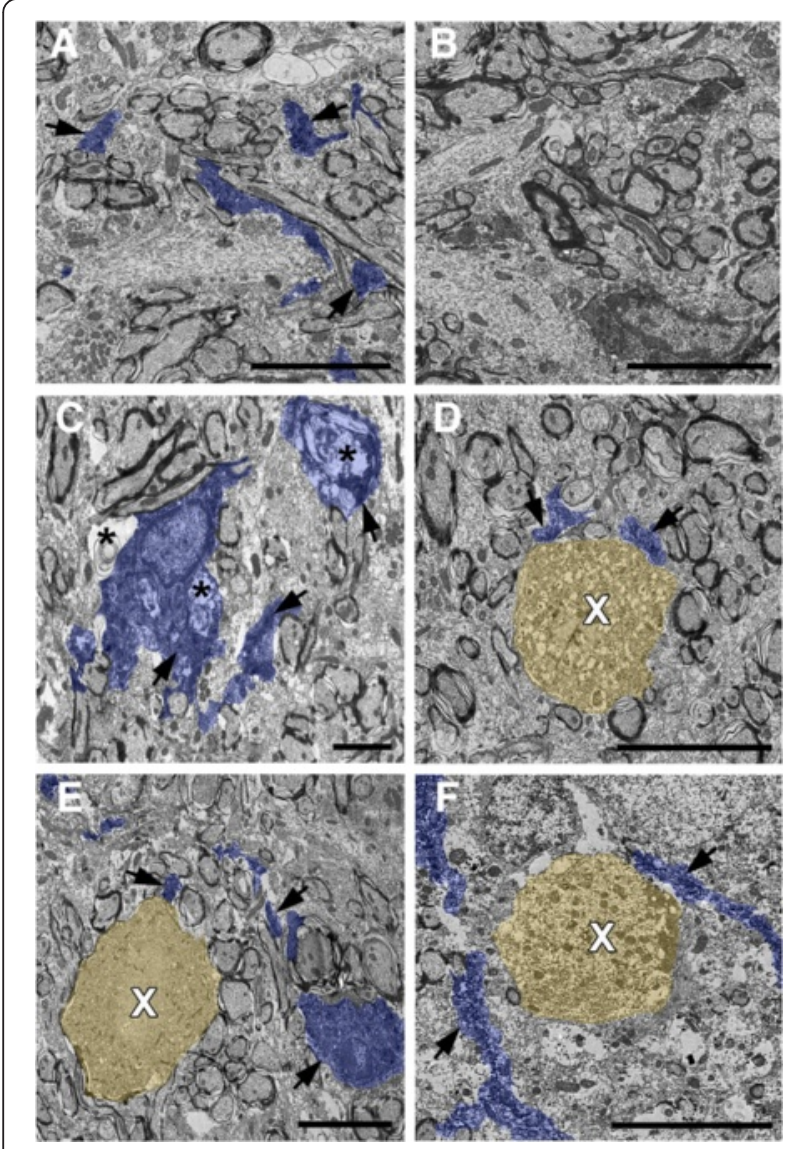

Fig. 8 Ultrastructural evidence of non-phagocytic microglial processes directly contacting proximal axonal swellings undergoing acute DAI. Representative electron micrographs of Iba-1 immuno-electronmicroscope-labeled microglia in the thalamus of sham ( $\mathbf{a}$ and $\mathbf{b}$ ) and injured (c-f) animals. Iba-1-labeled structures in $\mathbf{a}$ and $\mathbf{c}-\mathbf{f}$ are pseudocolored blue for clarity. Note that, microglia processes containing Iba-1 (arrows) within the thalamus of sham-injured animals (a and $\mathbf{b}$ ) are widely dispersed with no apparent association with axonal segments. Following CFPI, however, Iba-1+ microglial processes are much more prevalent. Some phagocytic microglia (c), containing material consistent with Wallerian degeneration (asterisks), are present following injury. These phagocytic microglia are primarily localized to areas of axonal damage/ Wallerian degeneration. $\mathbf{d - f}$ Microglial processes (pseudo-colored blue) associating with axonal swellings (pseudo-colored yellow), as identified using the common ultrastructural features observed previously for diffuse axonal injury, appeared most frequently as $\mathbf{d}$ Iba-1 immunolabeled (arrows) puncta adjacent to the axonal swelling (white X), however, some cases $\mathbf{e}$ and $\mathbf{f}$ allowed for the tracing of microglia processes over short distances. Importantly, the Iba-1+ microglia processes found associated with axonal swellings did not reveal ultrastructural changes consistent with phagocytosis. Scale bar: $5 \mu \mathrm{m}$

profiles and responses $[47,48]$, suggests that this association could be exploited in humans early in the posttraumatic period. Recent observations indicating similar associations between DAI and neuroinflammation in humans weeks to years following injury lend credence to this possibility [69]. Techniques utilizing PET imaging to visualize human neuroinflammation could be employed to assess areas sustaining acute DAI in TBI patients in a way never before possible. Additionally, alterations in neuroinflammatory biomarkers within the serum following TBI could also be used in conjunction with these imaging techniques to estimate the total burden of diffuse injury and better tailor treatment in the human population. Future studies exploring the temporal relationship between neuroinflammation and the pathological progression of DAI through to sub-acute and even chronic time points in this higher order micro pig model could prove invaluable in understanding the interplay between DAI and neuroinflammation in the gyrencephalic brain.

\section{Additional files}

Additional file 1: Processes of ramified microglia primarily pass over normal myelinated fibers in the sham thalamus. Movie representing 3D reconstructions of confocal z-stacks of thalamic sites from sham-injured animals, in which Dapi-labeled nuclei are in blue, $\mathrm{MBP}+$ myelinated axons are in red, and lba-1+ microglia are in white. Purple circles highlight contacts between microglial processes and normal myelinated fibers. Notice that while only a few microglial processes come in direct apposition with normal myelinated fibers, the majority of microglial processes that do contact myelinated fibers do not terminate at the axon; rather pass over the myelinated fiber. Scale bar: $10 \mu \mathrm{m}$. (MP4 $16442 \mathrm{~kb}$ )

Additional file 2: Processes of activated microglia terminate on proximal swellings of axons sustaining acute DAI. Movie representing 3D reconstructions of confocal $z$-stacks of thalamic sites from CFPI animals, in which Dapi-labeled nuclei are in blue, MBP+ myelinated axons are in red, APP + proximal axonal swellings are in green, and lba-1+ microglia are in white. Purple circles highlight contacts between microglial processes and APP+ proximal axonal swellings. Note that the majority of microglial processes that come into contact with APP+ axonal swellings terminate on the proximal swelling, indicating process convergence. Scale bar: $10 \mu \mathrm{m}$. (MP4 $12603 \mathrm{~kb}$ )

\section{Abbreviations}

ANOVA: analysis of variance; APP: amyloid precursor protein; cFPl: central fluid percussion injury; DAl: diffuse axonal injury; MABP: mean arterial blood pressure; $\mathrm{mTBl}$ : mild traumatic brain injury; $\mathrm{PaO}_{2}$ : arterial oxygen tension; $\mathrm{PaCO}_{2}$ : arterial carbon dioxide pressure.

\section{Competing interests}

The authors declare that they have no competing interests.

\section{Authors' contributions}

ADL carried out the microscopic and ultrastructural analyses, conceived, designed, and coordinated the study, and wrote the manuscript. MT carried out the micro pig surgeries and physiological monitoring/analysis and participated in the microglial activation analysis. SAW participated in the micro pig surgeries and the design of the microscopic and ultrastructural studies. JTP participated in the micro pig surgeries and the ultrastructural analyses, conceived of the study and participated in its design, and wrote the manuscript. All authors read and approved the final manuscript.

\section{Acknowledgements}

The authors would like to thank Dr. Scott Henderson for his expertise in microscopy and image analysis, Dr. Robert Hamm for his biostatistical assistance and knowledge as well as Drs. David Loane, Kevin Wang, Patrick Kochanek, and Michelle Block for invigorating scientific discussions and suggestions. We would also like to recognize Lynn Davis, Jesse Sims, Karen Gorse, Judy Williamson, Frances White, and Dr. Thomas Taetzsch for invaluable technical assistance. This work was performed as a component of the Operation Brain Trauma Therapy consortium, which is supported by U.S. Army grants W81XWH-10-1-0623 and WH81XWH-14-2-0018. Microscopy was 
performed at the VCU Department of Anatomy and Neurobiology Microscopy Facility, supported, in part, with funding from NIH-NINDS Center core grant 5P30NS047463.

\section{Author details}

${ }^{1}$ Department of Anatomy and Neurobiology, Virginia Commonwealth University Medical Center, P.O. Box 980709, Richmond, VA 23298, USA ${ }^{2}$ Advanced Medical Emergency and Critical Care Center, Yamaguchi University Hospital, Yamaguchi, Japan.

\section{Received: 27 July 2015 Accepted: 23 September 2015} Published online: 06 October 2015

\section{References}

1. Langlois JA, Rutland-Brown W, Wald MM. The epidemiology and impact of traumatic brain injury: a brief overview. J Head Trauma Rehabil. 2006;:21:375-8

2. Coronado VG, Xu L, Basavaraju SV, McGuire LC, Wald MM, Faul MD, et al. Surveillance for traumatic brain injury-related deaths_-United States, 19972007. MMWR Surveill Summ. 2011:60:1-32.

3. Koskinen S, Alaranta H. Traumatic brain injury in Finland 1991-2005: a nationwide register study of hospitalized and fatal TBI. Brain Inj. 2008;22:205-14.

4. Feigin VL, Theadom A, Barker-Collo S, Starkey NJ, McPherson K, Kahan M, et al. Incidence of traumatic brain injury in New Zealand: a population-based study. Lancet Neurol. 2013;12:53-64.

5. Barkhoudarian G, Hovda DA, Giza CC. The molecular pathophysiology of concussive brain injury. Clin Sports Med. 2011;30:33-48. vii-iii.

6. Farkas O, Povlishock JT. Cellular and subcellular change evoked by diffuse traumatic brain injury: a complex web of change extending far beyond focal damage. Prog Brain Res. 2007:161:43-59.

7. McGinn M, Povlishock J. Cellular and molecular mechanisms of injury and spontaneous recovery. In: Handbook of clinical neurology. 2015;127:67-87.

8. Hemphill MA, Dauth S, Yu CJ, Dabiri BE, Parker KK. Review traumatic brain injury and the neuronal microenvironment : a potential role for neuropathological mechanotransduction. Neuron. 2015;85:1177-92.

9. Greve MW, Zink BJ. Pathophysiology of traumatic brain injury. Mt Sinai J Med. 2009;76:97-104

10. Gennarelli TA, Thibault LE, Adams JH, Graham DI, Thompson CJ, Marcincin RP. Diffuse axonal injury and traumatic coma in the primate. Ann Neurol. 1982;12:564-74.

11. Povlishock JT. Pathobiology of traumatically induced axonal injury in animals and man. Ann Emerg Med. 1993;22:980-6.

12. Kelley BJ, Farkas O, Lifshitz J, Povlishock JT. Traumatic axonal injury in the perisomatic domain triggers ultrarapid secondary axotomy and Wallerian degeneration. Exp Neurol. 2006;198:350-60.

13. Smith DH, Meaney DF. Axonal damage in traumatic brain injury. Neurosci. 2000;6:483-95.

14. Adams JH, Graham DI, Murray LS, Scott G. Diffuse axonal injury due to nonmissile head injury in humans: an analysis of 45 cases. Ann Neurol. 1982:12:557-63.

15. Kraus MF, Susmaras T, Caughlin BP, Walker CJ, Sweeney JA, Little DM. White matter integrity and cognition in chronic traumatic brain injury: a diffusion tensor imaging study. Brain. 2007;130(Pt 10):2508-19.

16. Browne KD, Chen X-H, Meaney DF, Smith DH. Mild traumatic brain injury and diffuse axonal injury in swine. J Neurotrauma. 2011;28:1747-55.

17. Johnson VE, Stewart W, Smith DH. Axonal pathology in traumatic brain injury. Exp Neurol. 2013;246:35-43.

18. Scheid R, Walther K, Guthke T, Preul C, von Cramon DY. Cognitive sequelae of diffuse axonal injury. Arch Neurol. 2006;63:418-24.

19. Johnson VE, Stewart JE, Begbie FD, Trojanowski JQ, Smith DH, Stewart W. Inflammation and white matter degeneration persist for years after a single traumatic brain injury. Brain. 2013;136:28-42.

20. Loane DJ, Kumar A, Stoica B, Cabatbat R, Faden Al. Progressive neurodegeneration after experimental brain trauma: association with chronic microglial activation. J Neuropathol Exp Neurol. 2013:73:14-29.

21. Kumar A, Loane DJ. Neuroinflammation after traumatic brain injury: opportunities for therapeutic intervention. Brain Behav Immun. 2012;26:1191-201.

22. Kumar A, Stoica B, Sabirzhanov B, Burns MP, Faden Al, Loane DJ. Traumatic brain injury in aged animals increases lesion size and chronically alters microglial/macrophage classical and alternative activation states. Neurobiol Aging. 2013;34:1397-411.

23. Hinson HE, Rowell S, Schreiber M. Clinical evidence of inflammation driving secondary brain injury. J Trauma Acute Care Surg. 2015;78:184-91.

24. Bell MJ, Kochanek PM, Doughty L, Carcillo J, Adelson PD, Clark RSB, et al. Interleukin-6 and interleukin-10 in cerebrospinal fluid after severe traumatic brain injury in children. J Neurotrauma. 1997;14:451-7.

25. Taetzsch T, Levesque S, McGraw C, Brookins S, Luqa R, Bonini MG, et al. Redox regulation of NF-KB p50 and M1 polarization in microglia. Glia. 2015;63:423-40.

26. Byrnes KR, Loane DJ, Stoica B, Zhang J, Faden Al. Delayed mGluR5 activation limits neuroinflammation and neurodegeneration after traumatic brain injury. J Neuroinflammation. 2012;9:43.

27. Haynes SE, Hollopeter G, Yang G, Kurpius D, Dailey ME, Gan W-B, et al. The P2Y12 receptor regulates microglial activation by extracellular nucleotides. Nat Neurosci. 2006;9:1512-9.

28. Kyritsis N, Kizil C, Zocher S, Kroehne V, Kaslin J, Freudenreich D, et al. Acute inflammation initiates the regenerative response in the adult zebrafish brain. Science. 2012;338:1353-6.

29. Aguzzi A, Barres B, Bennett ML. Microglia: scapegoat, saboteur or something else? Inflammation. 2013:339:156-62.

30. Parkhurst CN, Yang G, Ninan I, Savas JN, Yates JR, Lafaille JJ, et al. Microglia promote learning-dependent synapse formation through brain-derived neurotrophic factor. Cell. 2013;155:1596-609.

31. Miyamoto A, Wake H, Moorhouse AJ, Nabekura J. Microglia and synapse interactions: fine tuning neural circuits and candidate molecules. Front Cell Neurosci. 2013;7(May):70.

32. Batchelor PE, Porritt MJ, Martinello P, Parish CL, Liberatore GT, Donnan G, et al. Macrophages and microglia produce local trophic gradients that stimulate axonal sprouting toward but not beyond the wound edge. Mol Cell Neurosci. 2002;21:436-53.

33. Venkatesan C, Chrzaszcz M, Choi N, Wainwright MS. Chronic upregulation of activated microglia immunoreactive for galectin-3/Mac-2 and nerve growth factor following diffuse axonal injury. J Neuroinflammation. 2010;7:32.

34. Jebelli J, Su W, Hopkins S, Pocock J, Garden GA. Glia: guardians, gluttons, or guides for the maintenance of neuronal connectivity? Ann N Y Acad Sci. 2015;1351:1-10.

35. Shitaka Y, Tran HT, Bennett RE, Sanchez L, Levy M, Dikranian K, et al. Repetitive closed-skull traumatic brain injury in mice causes persistent multifocal axonal injury and microglial reactivity. J Neuropathol Exp Neurol. 2012;70:551-67.

36. Wang J, Fox MA, Povlishock JT. Diffuse traumatic axonal injury in the optic nerve does not elicit retinal ganglion cell loss. J Neuropathol Exp Neurol. 2013;72:768-81.

37. Oehmichen $\mathrm{M}$, Theuerkauf I, Meißner C. Is traumatic axonal injury (Al) associated with an early microglial activation? Application of a doublelabeling technique for simultaneous detection of microglia and Al. Acta Neuropathol. 1999:97:491-4.

38. Gyoneva S, Ransohoff RM. Inflammatory reaction after traumatic brain injury: therapeutic potential of targeting cell-cell communication by chemokines. Trends Pharmacol Sci. 2015;1-10.

39. Kelley BJ, Lifshitz J, Povlishock JT. Neuroinflammatory responses after experimental diffuse traumatic brain injury. J Neuropathol Exp Neurol. 2007;66:989-1001.

40. Lawson L, Perry V, Gordon S. Heterogeneity in the distribution and morphology of microglia in the normal adult mouse brain. Neuroscience. 1990;39:151-70

41. McKay SM, Brooks DJ, Hu P, McLachlan EM. Distinct types of microglial activation in white and grey matter of rat lumbosacral cord after midthoracic spinal transection. J Neuropathol Exp Neurol. 2007;66:698-710.

42. Ogura K, Ogawa M, Yoshida M. Effects of ageing on microglia in the normal rat brain: immunohistochemical observations. Neuroreport. 1994;5:1224-6.

43. Seok J, Warren HS, Cuenca AG, Mindrinos MN, Baker HV, Xu W, et al. Genomic responses in mouse models poorly mimic human inflammatory diseases. Proc Natl Acad Sci U S A. 2013;110:3507-12.

44. Heinz S, Haehnel V, Karaghiosoff M, Schwarzfischer L, Müller M, Krause SW, et al. Species-specific regulation of toll-like receptor 3 genes in men and mice. J Biol Chem. 2003;278:21502-9.

45. Roshick C, Wood H, Caldwell HD, McClarty G. Comparison of gamma interferon-mediated antichlamydial defense mechanisms in human and mouse cells. Infect Immun. 2006;74:225-38. 
46. Mikaberidze A. Molecular mimicry and the generation of host defense protein diversity. Cell. 1993;72:823-6.

47. Fairbairn L, Kapetanovic R, Sester DP, Hume D. The mononuclear phagocyte system of the pig as a model for understanding human innate immunity and disease. J Leukoc Biol. 2011;89:855-71.

48. Wernersson R, Schierup MH, Jørgensen FG, Gorodkin J, Panitz F, Staerfeldt $\mathrm{H}-\mathrm{H}$, et al. Pigs in sequence space: a $0.66 \mathrm{X}$ coverage pig genome survey based on shotgun sequencing. BMC Genomics. 2005;6:70.

49. Anderson CV, Wood DM, Bigler ED, Blatter DD. Lesion volume, injury severity, and thalamic integrity following head injury. J Neurotrauma. 1996;13:59-65.

50. Schiff ND, Giacino JT, Kalmar K, Victor JD, Baker K, Gerber M, et al. Behavioural improvements with thalamic stimulation after severe traumatic brain injury. Nature. 2007;448:600-3.

51. Ramlackhansingh AF, Brooks DJ, Greenwood RJ, Bose SK, Turkheimer FE, Kinnunen KM, et al. Inflammation after trauma: microglial activation and traumatic brain injury. Ann Neurol. 2011;70:374-83.

52. Ross DT, Graham DI, Adams JH. Selective loss of neurons from the thalamic reticular nucleus following severe human head injury. J Neurotrauma. 1993;10:151-65.

53. Sherriff FE, Bridges LR, Sivaloganathan S. Early detection of axonal injury after human head trauma using immunocytochemistry for beta-amyloid precursor protein. Acta Neuropathol. 1994;87:55-62.

54. Gentleman SM, Nash MJ, Sweeting CJ, Graham DI, Roberts GW. Betaamyloid precursor protein (beta APP) as a marker for axonal injury after head injury. Neurosci Lett. 1993;160:139-44.

55. Dixon CE, Lyeth BG, Povlishock JT, Findling RL, Hamm RJ, Marmarou A, et al. A fluid percussion model of experimental brain injury in the rat. J Neurosurg. 1987;67:110-9.

56. Bove A. Diving medicine. Am J Respir Crit Care Med. 2014;189:1479-86.

57. Thomson L, Paton J. Oxygen toxicity. Paediatr Respir Rev. 2014;15:120-3.

58. Hånell A, Greer JE, McGinn MJ, Povlishock JT. Traumatic brain injury-induced axonal phenotypes react differently to treatment. Acta Neuropathol. 2014;129:317-32.

59. Wang J, Hamm RJ, Povlishock JT. Traumatic axonal injury in the optic nerve: evidence for axonal swelling, disconnection, dieback, and reorganization. J Neurotrauma. 2011;28:1185-98.

60. Christman CW, Grady MS, Walker SA, Holloway KL, Povlishock JT. Ultrastructural studies of diffuse axonal injury in humans. J Neurotrauma. 1994;11:173-86.

61. Eyo UB, Peng J, Swiatkowski P, Mukherjee A, Bispo A, Wu L-J. Neuronal hyperactivity recruits microglial processes via neuronal NMDA receptors and microglial P2Y12 receptors after status epilepticus. J Neurosci. 2014;34:10528-40.

62. Yamasaki R, Lu H, Butovsky O, Ohno N, Rietsch AM, Cialic R, et al. Differential roles of microglia and monocytes in the inflamed central nervous system. J Exp Med. 2014;211:1533-49.

63. Lafrenaye AD, Krahe TE, Povlishock JT. Moderately elevated intracranial pressure after diffuse traumatic brain injury is associated with exacerbated neuronal pathology and behavioral morbidity in the rat. J Cereb Blood Flow Metab. 2014;34:1628-36.

64. Coughlin JM, Wang Y, Munro C, Ma S, Yue C, Chen S, et al. Neuroinflammation and brain atrophy in former NFL players: an in vivo multimodal imaging pilot study. Neurobiol Dis. 2015;74:58-65.

65. Kondo Y, Adams JM, Vanier MT, Duncan ID. Macrophages counteract demyelination in a mouse model of globoid cell leukodystrophy. J Neurosci. 2011;31:3610-24.

66. Tönges L, Günther R, Suhr M, Jansen J, Balck A, Saal KA, et al. Rho kinase inhibition modulates microglia activation and improves survival in a model of amyotrophic lateral sclerosis. Glia. 2014;62:217-32.

67. Nemeth CL, Reddy R, Bekhbat M, Bailey J, Neigh GN. Microglial activation occurs in the absence of anxiety-like behavior following microembolic stroke in female, but not male, rats. J Neuroinflammation. 2014;11:174.

68. Howell OW, Rundle JL, Garg A, Komada M, Brophy PJ, Reynolds R. Activated microglia mediate axoglial disruption that contributes to axonal injury in multiple sclerosis. J Neuropathol Exp Neurol. 2010;69:1017-33.

69. Ryu J, Horkayne-Szakaly I, Xu L, Pletnikova O, Leri F, Eberhart C, et al. The problem of axonal injury in the brains of veterans with histories of blast exposure. Acta Neuropathol Commun. 2014;2:1-14.

70. Davalos D, Grutzendler J, Yang G, Kim JV, Zuo Y, Jung S, et al. ATP mediates rapid microglial response to local brain injury in vivo. Nat Neurosci. 2005;8:752-8.
71. Schafer DP, Lehrman EK, Stevens B. The "quad-partite" synapse: microgliasynapse interactions in the developing and mature CNS. Glia. 2013;61:24-36.

72. Neumann H, Kotter MR, Franklin RJM. Debris clearance by microglia: an essential link between degeneration and regeneration. Brain. 2009;132(Pt 2):288-95.

73. Baalman K, Marin M, Ho TS-Y, Godoy M, Cherian L, Robertson C, et al. Axon initial segment-associated microglia. J Neurosci. 2015;35:2283-92.

74. Nimmerjahn A, Kirchhoff F, Helmchen F. Resting microglial cells are highly dynamic surveillants of brain parenchyma in vivo-supporting online material. Science. 2005;308:1314-9.

75. Damani MR, Zhao L, Fontainhas AM, Amaral J, Fariss RN, Wong WT. Agerelated alterations in the dynamic behavior of microglia. Aging Cell. 2011;10:263-76.

76. Wake H, Moorhouse AJ, Miyamoto A, Nabekura J. Microglia: actively surveying and shaping neuronal circuit structure and function. Trends Neurosci. 2013;36:209-17.

77. Krabbe G, Matyash V, Pannasch U, Mamer L, Boddeke HWGM, Kettenmann $H$. Activation of serotonin receptors promotes microglial injury-induced motility but attenuates phagocytic activity. Brain Behav Immun. 2012;26:419-28

78. Eyo UB, Gu N, De S, Dong H, Richardson JR, Wu L-J. Modulation of microglial process convergence toward neuronal dendrites by extracellular calcium. J Neurosci. 2015;35:2417-22.

79. Chen Z, Jalabi W, Hu W, Park H-J, Gale JT, Kidd GJ, et al. Microglial displacement of inhibitory synapses provides neuroprotection in the adult brain. Nat Commun. 2014;5(July):4486-98.

80. Lifshitz J, Kelley BJ, Povlishock JT. Perisomatic thalamic axotomy after diffuse traumatic brain injury is associated with atrophy rather than cell death. J Neuropathol Exp Neurol. 2007;66:218-29.

81. Greer JE, McGinn MJ, Povlishock JT. Diffuse traumatic axonal injury in the mouse induces atrophy, c-Jun activation, and axonal outgrowth in the axotomized neuronal population. J Neurosci. 2011;31:5089-105.

82. Grady MS, MCLaughlin MR, Christman CW, Valadka AB, Fligner CL, Povlishock JT. The use of antibodies targeted against the neurofilament subunits for the detection of diffuse axonal injury in humans. J Neuropathol Exp Neurol. 1993;52:143-52.

83. Wolf JA, Stys PK, Lusardi T, Meaney D, Smith DH. Traumatic axonal injury induces calcium influx modulated by tetrodotoxin-sensitive sodium channels. J Neurosci. 2001;21:1923-30.

84. LoPachin RM, Lehning EJ. Mechanism of calcium entry during axon injury and degeneration. Toxicol Appl Pharmacol. 1997;143:233-44.

85. Cohen AS, Pfister BJ, Schwarzbach E, Grady MS, Goforth PB, Satin LS. Injuryinduced alterations in CNS electrophysiology. Prog Brain Res. 2007;161:143-69.

86. Greer JE, Povlishock JT, Jacobs KM. Electrophysiological abnormalities in both axotomized and nonaxotomized pyramidal neurons following mild traumatic brain injury. J Neurosci. 2012;32:6682-7.

87. Reeves TM, Phillips LL, Povlishock JT. Myelinated and unmyelinated axons of the corpus callosum differ in vulnerability and functional recovery following traumatic brain injury. Exp Neurol. 2005;196:126-37.

88. Girard S, Brough D, Lopez-Castejon G, Giles J, Rothwell NJ, Allan SM. Microglia and macrophages differentially modulate cell death after brain injury caused by oxygen-glucose deprivation in organotypic brain slices. Glia. 2013;61:813-24.

89. Tang $Y$, Le W. Differential roles of $M 1$ and $M 2$ microglia in neurodegenerative diseases. Mol Neurobiol 2015. Epub ahead of print.

90. Tam WY, Ma CHE. Bipolar/rod-shaped microglia are proliferating microglia with distinct M1/M2 phenotypes. Sci Rep. 2014;4:7279.

91. Martinez FO, Helming L, Milde R, Varin A, Melgert BN, Draijer C, et al. Genetic programs expressed in resting and IL-4 alternatively activated mouse and human macrophages: Similarities and differences. Blood. 2013;121:57-69.

92. Karve IP, Taylor JM, Crack PJ. The contribution of astrocytes and microglia to traumatic brain injury. Br J Pharmacol 2015. Epub ahead of print.

93. Wang G, Shi $Y$, Jiang $X$, Leak RK, Hu X, Wu Y, et al. HDAC inhibition prevents white matter injury by modulating microglia/macrophage polarization through the GSK3ß/PTEN/Akt axis. PNAS. 2015;112:2853-58.

94. Banati RB. Visualising microglial activation in vivo. Glia. 2002;40:206-17. 